\title{
Effect of esomeprazole on lipid profile in patients with peptic ulcer
}

\author{
Eman Abdullah¹, Sarraa Dhiaa', Khalaf Saleh², Marwan Merkhan ${ }^{3}$ \\ 1 College of Pharmacy, University of Mosul, Mosul, Iraq \\ 2 Ibn Sina Teaching Hospital, Mosul, Iraq \\ 3 College of Pharmacy, Ninevah University, Mosul, Iraq \\ Corresponding author: Marwan Merkhan (marwan.mohammed@uoninevah.edu.iq)
}

Received 17 June 2021 • Accepted 8 August 2021 • Published 17 August 2021

Citation: Abdullah E, Dhiaa S, Saleh K, Merkhan M (2021) Effect of esomeprazole on lipid profile in patients with peptic ulcer. Pharmacia 68(3): 613-617. https://doi.org/10.3897/pharmacia.68.e70292

\begin{abstract}
Esomeprazole; a newly introduced PPI has been widely prescribed by healthcare providers due to its improved pharmacokinetic profile. Most users could have other diseases and the PPIs are indicated as acid-suppressor to minimize gastric side effects of polypharmacy. A high percentage of users could suffer from cardiovascular diseases and lipid dysmetabolism. Hence, this study was designed to determine the impact of long-term use of esomeprazole on lipid profile in a normal subject other than having peptic ulcer for which esomeprazole has been indicated. Results confirmed that esomeprazole reduced triglyceride and HDL levels and elevated total cholesterol level and correspondingly LDL level was elevated, however, no effect was noticed with VLDL. To sum up, esomeprazole impaired lipid metabolism in apparently normal healthy individuals apart from having peptic ulcer for which the esomeprazole was indicated, this finding rise a caution during prescribing esomeprazole for the patient with multiple diseases and polypharmacy including cardiovascular ailments.
\end{abstract}

\section{Keywords}

Cholesterol, Esomeprazole, Lipoprotein, Triglycerides

\section{Introduction}

Tissue injury due to whatever stress-inducers might result of a bi-armed injury; oxidative stress and inflammation (Shiraev and Bullen 2018). The production of free radicals and exhaustion of local antioxidant micronutrients in the battle zone demonstrating oxidative burden on the local environment resulting in progression of tissue injury and further advancement of the disease status (Wetscher et al. 1995; Drake et al. 1998). It has been confirmed that intestinal infection with Helicobacter pylori is associated with a storm of free radical production and a laboratory test of a patient with gastritis revealed increased radioactivity at gastric lumen using chemiluminescent reagent indicating free radical accumulation and the study has confirmed that the luminescence activity decreased following bacterial eradication (Drake et al. 1998). Additionally, in vitro studies confirmed that Helicobacter pylori reduced glutathione level significantly confirming that Helicobacter pylori contribute to overall local oxidative stress (Wetscher et al. 1995).

Acid suppression strategy is a cornerstone for peptic ulcer management together with antibiotics used to eradicate bacterial gastritis (Aamir and Ali 2019). However, some of these agents are preferred over others, due to their efficacy or safety profile (Koch et al. 2004; Shiraev and Bullen 2018). For instance, PPIs are prescribed favorably due to their efficacy over H2-blocker or acid-neutralizing capacity. Moreover, new studies 
confirmed that PPIs demonstrate a higher healing rate and reduced recurrence chances compared to other acid-counteracting agents (Huang and Hunt 1996; Farley et al. 2000). Nonetheless, a combination with antibacterial agents should be fulfilled regardless of the acid-suppressing regimen used (Huang and Hunt 1996; Almulathanon et al. 2021).

PPIs are the most commonly prescribed acid-suppressing agent in private clinics and hospital settings (Heidelbaugh et al. 2012; Arora et al. 2016). The group consist of 5 members, these include, omeprazole (prototype), lansoprazole, esomeprazole, rabiprazole, and pantoprazole (Aamir and Ali 2019). They are sharing the mode of action through blocking the proton pump (H/K-ATPase enzyme) and thereby blocking HCL secretion (Koch et al. 2004). They are used for peptic ulcers, Drug- or stress-induced ulcers (Ji et al. 2012; Aamir and Ali 2019). Upon introduction to the market, their use has quickly built up over few years. The initially reported adverse effect profile was tolerable, nevertheless, later on, the reported side effects extended to include nephrotoxicity due to precipitation of insoluble parent drugs and/or their metabolite (Aronson 2016; Xie et al. 2017; Morschel et al. 2018), moreover, PPIs side effects on the heart has been reported with lacking explanation of the mode of action (Koch et al. 2004). Herein, we are trying to demonstrate the effect of esomeprazole on lipid parameters if any. These types of studies could clarify the mechanism of side effects on the cardiovascular system.

\section{Materials and methods}

To conduct this study, patients using esomeprazole were recruited from outpatients clinic and following careful examination and medical history, only those patients with clear medical records of no history of chronic diseases were enrolled in the study. A total of 55 subjects (29 apparently control healthy and 26 peptic ulcer patients) were recruited from outpatients clinics and enrolled in the present study. For patients with ulcer to be included in the study, should be healthy from systemic diseases except for ulcer and should be on esomeprazole continuously for at least 2 months (40 mg per day; Nexium, AstraZeneca, UK). Pregnant women, lactating mothers, and patients with any sort of chronic diseases were excluded from the study or those patients with polypharmacy. The demographic parameters are mentioned below (Table 1). A consent of agreement has been taken from all patients.

Table 1. Demographic parameters for the studied groups.

\begin{tabular}{lcc}
\hline \multicolumn{1}{c}{ Parameters } & \multicolumn{2}{c}{ Studied Group $(\mathbf{n}=\mathbf{5 5})$} \\
\cline { 2 - 3 } & Control $(\mathbf{n}=\mathbf{2 9})$ & Esomeprazole $(\mathbf{n}=\mathbf{2 6})$ \\
\hline Age (years) & $41.54 \pm 11.05$ & $35.14 \pm 16.6$ \\
Weight $(\mathbf{k g})$ & $70.04 \pm 5.16$ & $65.28 \pm 13.06$ \\
Duration of treatment & - & 2 months \\
Gender (Male/Female) & $17 / 12$ & $13 / 13$ \\
\hline
\end{tabular}

For laboratory tests, venous blood was withdrawn from 10-hour fasting patients and control subjects. Blood was centrifuged and serum collected and froze for laboratory analysis. Upon analysis, frozen samples were thawed and analyzed. The analysis was performed using enzymatic methods (Cobas 6000, Roche-Diagnostic, Swiss) measuring TC, TG, and HDL-c. Initially, the serum samples were primed with magnesium and phosphotungstic acid to precipitate all the present components in plasma except TC-associated lipoproteins. This step was then followed by an enzymatic reaction producing formazan dye. The concentration of TC is reciprocal to the intensity of the dye produced which has been quantified spectrophotometrically.

Triglyceride measurement was done based on the above-mentioned enzymatic reaction. The intensity of formazan dye is reciprocally proportional to the glycerol produced from the hydrolysis of TG according to the manufacture's instruction. To calculate LDL, the Friedewald formula (LDL $=\mathrm{TC}-\mathrm{HDL}-\mathrm{TG} / 5)$ was used. vLDL and non-HDL are automatically calculated by Cobas6000 instrument.

Similarly, cholesterol was measured following manufacturer instruction, serum samples were treated with PBS and the cholesterol in these samples was de-esterified enzymatically to cholesterol and free fatty acids. The resultant cholesterol was then oxidized to cholestenone by cholesterol dehydrogenase catalyzed by nicotinamide-adenine dinucleotide (NAD). Cholestenone reduced to formazan dye by diaphorase enzyme and $\mathrm{NADH}$ by redox reactions and the formazan dye intensity reciprocally correlates to cholesterol present in the samples (Fig. 1).

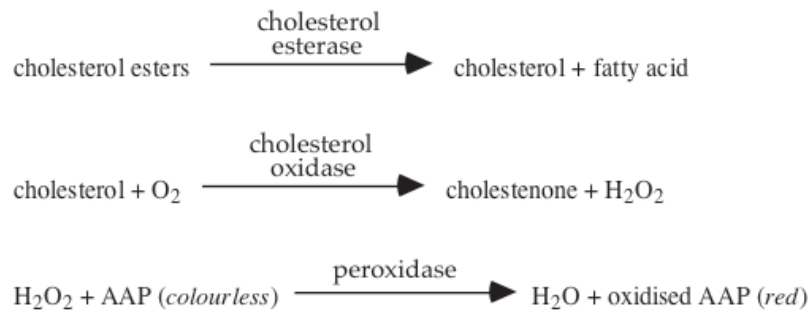

Figure 1. Steps of cholesterol redox reaction for quantification.

\section{Results and discussion}

The present study confirmed that long-term use of esomeprazole has resulted in impairment of lipid metabolism in peptic ulcer patients. The result showed that using esomeprazole in an apparently healthy individual (free from systemic diseases) has led to a significant $(\mathrm{p}<0.001)$ reduction of plasma levels $(\mathrm{mg} / \mathrm{dl})$ of TG and HDL(97.21 $\pm 41.72 ; 36.43 \pm 10.02)$ as compared to the control group (165.42 $\pm 24.56 ; 96.31 \pm 22.84)$ alongside significant $(\mathrm{p}<0.001)$ elevation of TC in esomeprazole group $(158.07 \pm 42.04)$ compared to control group(117.19 \pm 37.68$)$ (Fig. 2).

Correspondingly, the calculated parameters were reciprocally changed. Esomeprazole showed no effect on vLDL, however, it has significantly $(\mathrm{p}<0.001)$ elevated LDL and 


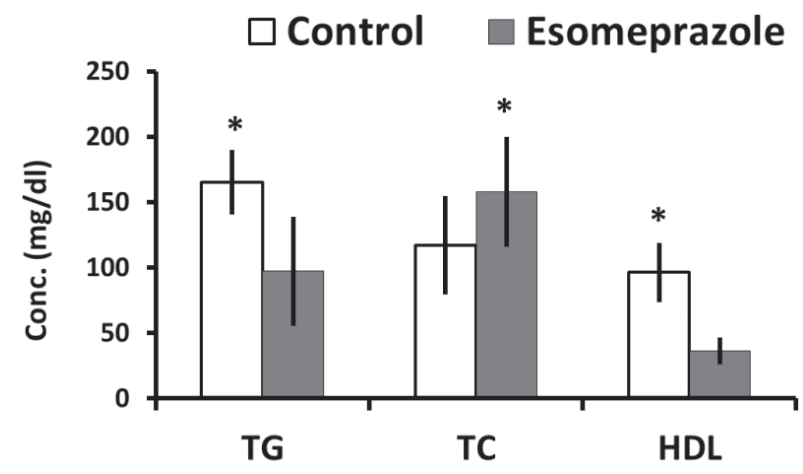

Figure 2. Esomeprazole impaired lipid metabolism in patients with peptic ulcer. Esomeprazole significantly decreased TG and $\mathrm{HDL}$ and significantly elevated TC. Data expressed as mean \pm SD, $\mathrm{p}<0.001, \mathrm{TG}=$ triglycerides, $\mathrm{TC}=$ total cholesterol, $\mathrm{HDL}=$ high density lipoprotein.

non-HDL $(102.34 \pm 35.38 ; 121.64 \pm 32.02)$ as compared to the control group $(44.58 \pm 13.87 ; 20.88 \pm 14.84)$ (Fig. 3).

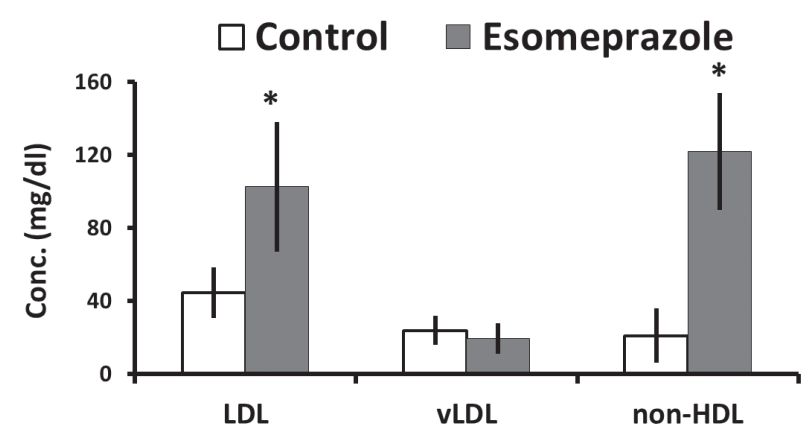

Figure 3. Esomeprazole modulated calculated hyperlipidemic indicators in patients with peptic ulcers. Esomeprazole significantly elevated LDL and non-HDL and has non-significant effects on vLDL. Data expressed as mean \pm SD, $p<0.001, \mathrm{LDL}=$ Low density lipoprotein, vLDL = very low-density lipoprotein, non-HDL = non-high density lipoprotein.

Previous studies focused on the safety profile of PPIs were reported increased morbidity and mortality in patients using PPIs, nevertheless, these studies were relating such actions to the interaction between PPIs and clopidogrel (antiplatelet agent) (Focks et al. 2013; Shiraev and Bullen 2018). Clopidogrel is structurally thioenopyridine, it binds to platelet surface receptor (P2Y12-receptor) preventing platelet aggregation (Shiraev and Bullen 2018); an action of highly importance in vascular diseases. The activation of clopidogrel is done in a liver enzyme (CYP2C19) resulting in the bioactivation of $15 \%$ of the administered dose, however, this hepatic metabolism in the liver is a sharing pathway involved in the catabolism of other drugs, including PPIs (Dean 2012). Therefore, a competition between PPIs and clopidogrel on enzyme has associated with reduced activation of clopidogrel increasing the cardiovascular risk of stroke. This explanation were previously dependent by researchers to clarify the increased morbidity and mortality rate associated with using PPIs in patients already on clopidogrel therapy (Focks et al. 2013), therefore, it was recommended to shift those patients to use either pantoprazole or $\mathrm{H} 2$-blockers for a gastric problem to avoid such interaction; pantoprazole is the only PPIs which doesn't involve in such interaction (Shiraev and Bullen 2018).

The above explanation was declined following demonstration of the same problem of PPIs use in inpatient with cardiovascular disease on ticagrelor, this later drug has no hepatic metabolism (Goodman et al. 2012). Therefore, scientists were looking for an alternative explanation. This may further confirm that lipid metabolism might be the cause of these abnormalities.

A study conducted on laboratory animals by Aamir et al. 2019 (Aamir and Ali 2019), screening the effect of PPIs (omeprazole, esomeprazole, and pantoprazole) on lipid dysmetabolism. The researcher has demonstrated that these PPIs significantly reduced HDL and elevated TC and TG, an action which is in the line with our result apart from TG showing reverse effects with our study (Fig. 4). This study was conducted on a healthy animal which means that PPIs impair lipid metabolism in normal healthy subjects.

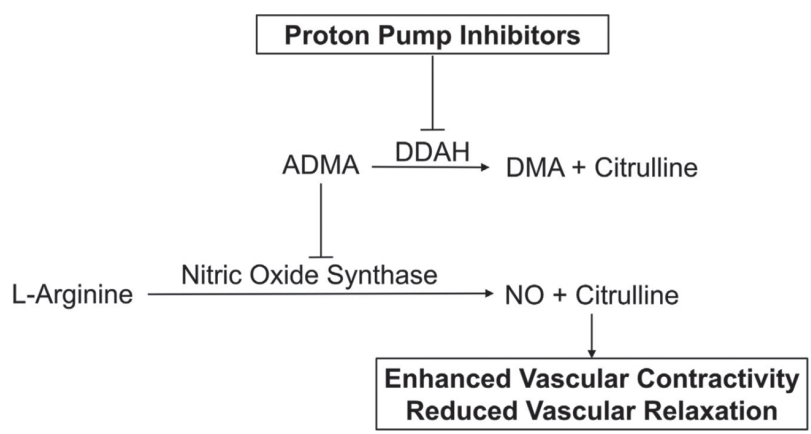

Figure 4. Mechanism of PPIs modulated vasculature.

Nevertheless, the explanation is still incomplete and further investigation is required to confirm the responsible enzymes or pathways about such effects. A separate study done by Ghebremariam et al. 2013 (Ghebremariam et al. 2013), demonstrated an impaired action of enzymes responsible for NO production resulting in loss of natural control of vascular contractility and consequently might be responsible for cardiac events associate with PPIs (Fig. 4). Correspondingly, Yepuri et al. 2016 (Yepuri et al. 2016), has reported that PPIs modulated lysosomal enzyme responsible for regulation of waste product catabolism; an action which is associated with vascular events.

Albeit that PPIs have modulated lipid in our study, the boundaries of action are still unclear and further detailed studies are to be initiated. In contrast to our findings, few researchers have confirmed that using PPIs has improved glycemic control and they explained that in term of modulated plasma gastrin level following administration of PPIs (Crouch et al. 2012; Takebayashi 2015), additionally, a retrospective study conducted by Barkas et al. 2015 (Barkas et al. 2015), revealed that PPIs boosted the hypolipidemic action of statins and esomeprazole was part of the study. The researcher has explained that in term of the ability of 
PPIs in modest suppression of liver enzyme (CYP3A4) apart from esomeprazole and omeprazole considered as strong inhibitors of CYP2C19; taking into consideration that the study has shown no changes in liver enzyme so as not to be involved as a contributory parameter. This variation together with structural variation might explain the differences between PPIs (Fig. 5).

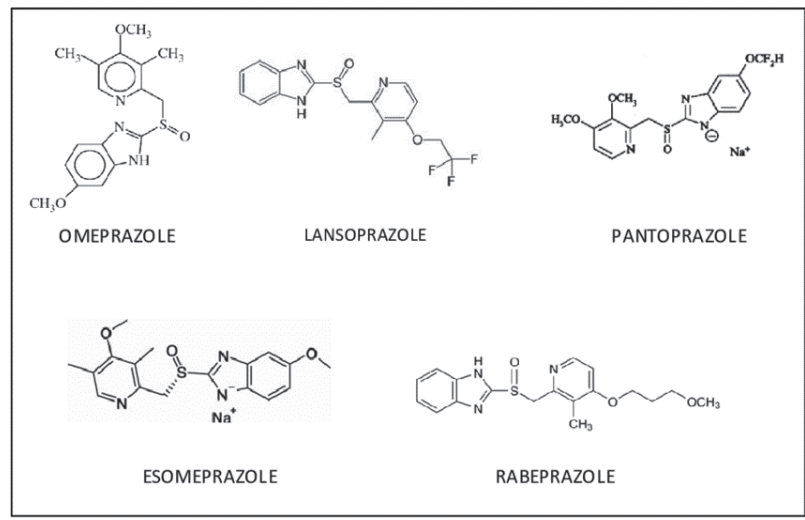

Figure 5. Structures of PPIs.

\section{References}

Aamir K, Arain AA, Tunio AG, Rasheed K, Soomro UA, Memon RA (2019) Do ppis affect serum lipids? A pilot study in rabbit model. Indo American Journal Of Pharmaceutical Sciences 6(2): 3060-3063.

Almulathanon AA, Mohammad JA, Fathi FH (2021) Comparative effects of metformin and glibenclamide on the redox balance in type 2 diabetic patients. Pharmacia 68: 327-332. https://doi.org/10.3897/ pharmacia.68.e63365

Arora P, Gupta A, Golzy M, Patel N, Carter RL, Jalal K, Lohr JW (2016) Proton pump inhibitors are associated with increased risk of development of chronic kidney disease. BMC nephrology 17(1): 1-8 https://doi.org/10.1186/s12882-016-0325-4

Barkas F, Elisaf M, Rizos CV, Klouras E, Kostapanos MS, Liberopoulos E (2015) Proton pump inhibitors and statins: a possible interaction that favors low-density lipoprotein cholesterol reduction? Hippokratia 19(4): 332-337.

Crouch MA, Mefford IN, Wade EU (2012) Proton pump inhibitor therapy associated with lower glycosylated hemoglobin levels in type $2 \mathrm{di}$ abetes. The Journal of the American Board of Family Medicine 25(1): 50-54. https://doi.org/10.3122/jabfm.2012.01.100161

Dean L (2012) Clopidogrel therapy and CYP 2C19 genotype. Medical genetics summaries. National Center for Biotechnology Information.

Drake IM, Mapstone NP, Schorah CJ, White KLM, Chalmers DM, Dixon MF, Axon ATR (1998) Reactive oxygen species activity and lipid peroxidation inHelicobacter pylori associated gastritis: relation to gastric mucosal ascorbic acid concentrations and effect of H pylori eradication. Gut 42(6): 768-771. https://doi.org/10.1136/ gut.42.6.768

Farley A, Wruble LD, Humphries TJ (2000) Rabeprazole versus ranitidine for the treatment of erosive gastroesophageal reflux disease:

\section{Conclusion}

The present study concluded that esomeprazole use impaired lipid metabolism by demonstrating modulated lipid parameters. These findings ensure using alternative safer PPIs than esomeprazole with lower lipid interaction to avoid precipitation of hyperlipidemia or accentuate the condition if hypercholesterolemia already present. We do recommend lipid profile tests for all high-risk patients to exclude those with impaired lipid values from esomeprazole indication.

\section{Acknowledgements}

The authors are very grateful to the University of Mosul/ College of Pharmacy and University of Ninevah/ College of Pharmacy for their provided facilities, which helped to improve the quality of this work. Thanks are also in order for the scientific committee in the department of pharmacology in the College of Pharmacy/University of Mosul. a double-blind, randomized clinical trial. The American journal of Gastroenterology 95(8): 1894-1899. https://doi.org/10.1111/j.15720241.2000.02233.x

Focks JJ, Brouwer MA, van Oijen MG, Lanas A, Bhatt DL, Verheugt FW (2013) Concomitant use of clopidogrel and proton pump inhibitors: impact on platelet function and clinical outcome - a systematic review. Heart 99(8): 520-527. https://doi.org/10.1136/heartjnl-2012-302371

Ghebremariam YT, LePendu P, Lee JC, Erlanson DA, Slaviero A, Shah NH, Leiper J, Cooke JP (2013) Unexpected effect of proton pump inhibitors: elevation of the cardiovascular risk factor asymmetric dimethylarginine. Circulation 128(8): 845-853. https://doi. org/10.1161/CIRCULATIONAHA.113.003602

Goodman SG, Clare R, Pieper KS, Nicolau JC, Storey RF, Cantor WJ, Mahaffey KW, Angiolillo DJ, Husted S, Cannon CP, James SK, Kilhamn J, Steg PG, Harrington RA, Wallentin L (2012) Association of proton pump inhibitor use on cardiovascular outcomes with clopidogrel and ticagrelor: insights from the platelet inhibition and patient outcomes trial. Circulation 125(8): 978-986. https://doi.org/10.1161/ CIRCULATIONAHA.111.032912

Heidelbaugh JJ, Kim AH, Chang R, Walker PC (2012) Overutilization of proton-pump inhibitors: what the clinician needs to know. Therapeutic advances in gastroenterology 5(4): 219-232. https://doi. org/10.1177/1756283X12437358

Huang JQ, Hunt RH (1996) pH, healing rate and symptom relief in acid-related diseases. The Yale Journal of Biology and Medicine 69(2): 159-174.

Ji CX, Fan DS, Li W, Guo L, Liang ZL, Xu RM, Zhang JJ (2012) Evaluation of the anti-ulcerogenic activity of the antidepressants duloxetine, amitriptyline, fluoxetine and mirtazapine in different models 
of experimental gastric ulcer in rats. European Journal of Pharmacology 691(1-3): 46-51. https://doi.org/10.1016/j.ejphar.2012.06.041 Koch TR, Petro A, Darrabie M, Opara EC (2004) Effect of the H, KATPase inhibitor, esomeprazole magnesium, on gut total antioxidant capacity in mice. The Journal of Nutritional Biochemistry 15(9): 522-526. https://doi.org/10.1016/j.jnutbio.2004.03.003

Morschel CF, Mafra D, Eduardo JCC (2018) The relationship between proton pump inhibitors and renal disease. Brazilian Journal of Nephrology 40(3): 301-306. https://doi.org/10.1590/2175-8239-jbn-2018-0021

Shiraev TP, Bullen A (2018) Proton pump inhibitors and cardiovascular events: a systematic review. Heart, Lung and Circulation 27(4): 443-450. https://doi.org/10.1016/j.hlc.2017.10.020

Takebayashi K, Inukai $\mathrm{T}$ (2015) Effect of proton pump inhibitors on glycemic control in patients with diabetes. World Journal of Diabetes 6(10): 1122-1131. https://doi.org/10.4239/wjd. v6.i10.1122

Wetscher GJ, Hinder RA, Bagchi D, Hinder PR, Bagchi M, Perdikis G, McGinn T (1995) Reflux esophagitis in humans is mediated by oxygen-derived free radicals. The American Journal of Surgery 170(6): 552-557. https://doi.org/10.1016/S0002-9610(99)80014-2

Xie Y, Bowe B, Li T, Xian H, Yan Y, Al-Aly Z (2017) Long-term kidney outcomes among users of proton pump inhibitors without intervening acute kidney injury. Kidney International 91(6): 1482-1494. https://doi.org/10.1016/j.kint.2016.12.021

Yepuri G, Sukhovershin R, Nazari-Shafti TZ, Petrascheck M, Ghebre YT, Cooke JP (2016) Proton pump inhibitors accelerate endothelial senescence. Circulation Research 118(12): e36-e42. https://doi. org/10.1161/CIRCRESAHA.116.308807 